\title{
Providing care for older adults in the Emergency Department: expert clinical recommendations from the European Task Force on Geriatric Emergency Medicine
}

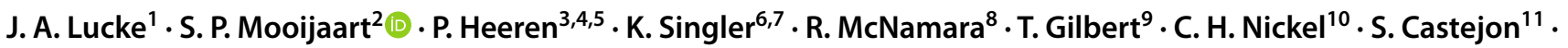 \\ A. Mitchell ${ }^{12} \cdot$ V. Mezera ${ }^{13} \cdot$ L. Van der Linden ${ }^{14,15}$. S. E. Lim ${ }^{16}$ - A. Thaur ${ }^{17} \cdot$ M. A. Karamercan ${ }^{18} \cdot$ L. C. Blomaard $^{2}$.

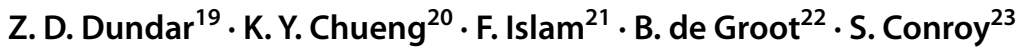

Received: 18 September 2021 / Accepted: 20 October 2021 / Published online: 5 November 2021

(c) The Author(s), under exclusive licence to European Geriatric Medicine Society 2021

\section{Key summary points}

Aim The aim was to develop expert clinical recommendations on Geriatric Emergency Medicine to be disseminated across Europe.

Findings Eight posters with expert clinical guidelines on the most important topics in Geriatric Emergency Medicine are now available through https://posters.geriemeurope.eu/.

Message Expert clinical recommendations for Geriatric Emergency Medicine in Europe were created and are ready for dissemination across Europe.

J. A. Lucke, S. P. Mooijaart, B. de Groot and S. Conroy contributed equally to this paper.

\section{J. A. Lucke}

jlucke@spaarnegasthuis.nl

1 Department of Emergency Medicine, Spaarne Gasthuis, Haarlem, The Netherlands

2 Department of Internal Medicine, Section on Gerontology and Geriatrics, Leiden University Medical Center, Leiden, The Netherlands

3 Department of Public Health and Primary Care, Academic Centre for Nursing and Midwifery, KU Leuven, Leuven, Belgium

4 Department of Geriatric Medicine, University Hospitals Leuven, Leuven, Belgium

5 Research Foundation Flanders, Brussels, Belgium

6 Department of Geriatrics, Klinikum Nürnberg, Paracelsus Private, Medical University, Nuremberg, Germany

7 Institute for Biomedicine of Ageing, Friedrich-Alexander-Uni versity, Erlangen-Nuremberg, Erlangen, Germany

8 Department of Emergency Medicine, St. Vincent's University Hospital, Dublin, Ireland

9 Department of Geriatric Medicine, Lyon-Sud University Hospital, Lyon, France

10 Emergency Medicine, University Hospital Basel, Basel, Switzerland
11 Department of Geriatrics and Palliative Care, Consorci Sanitari de Terrassa, Terrassa, Barcelona, Spain

12 Department of Emergency Medicine, Sligo University Hospital, Sligo, Ireland

13 Geriatric Center, Pardubice Hospital, Pardubice, Czech Republic

14 Pharmacy Department, University Hospitals Leuven, Leuven, Belgium

15 Department of Pharmaceutical and Pharmacological Sciences, KU Leuven, Leuven, Belgium

16 Academic Geriatric Medicine, University of Southampton, Southampton, UK

17 Department of Emergency Medicine, Guy's and St.Thomas' NHS Foundation Trust, London, UK

18 Department of Emergency Medicine, Gazi University School of Medicine, Ankara, Turkey

19 Department of Emergency Medicine, Necmettin Erbakan University Meram Faculty of Medicine, Konya, Turkey

20 Accident and Emergency Department, United Christian Hospital, Kwun Tong, Hong Kong

21 Department of Public Health and Primary Care, KU Leuven, Leuven, Belgium

22 Department of Emergency Medicine, Leiden University Medical Center, Leiden, The Netherlands

23 Geriatric Medicine, MRC Unit for Lifelong Health and Ageing at UCL, 5th Floor, 1-19 Torrington Place, London WC1E 7HB, UK 


\section{Abstract}

Purpose Despite the rapidly expanding knowledge in the field of Geriatric Emergency Medicine in Europe, widespread implementation of change is still lacking. Many opportunities in everyday clinical care are missed to improve care for this susceptible and growing patient group. The aim was to develop expert clinical recommendations on Geriatric Emergency Medicine to be disseminated across Europe.

Methods A group of multi-disciplinary experts in the field of Geriatric Emergency Medicine in Europe was assembled. Using a modified Delphi procedure, a prioritized list of topics related to Geriatric Emergency Medicine was created. Next, a multi-disciplinary group of nurses, geriatricians and emergency physicians performed a review of recent guidelines and literature to create recommendations. These recommendations were voted upon by a group of experts and placed on visually attractive posters. The expert group identified the following eight subject areas to develop expert recommendations on: Comprehensive Geriatric Assessment in the Emergency Department (ED), age/frailty adjusted risk stratification, delirium and cognitive impairment, medication reviews in the ED for older adults, family involvement, ED environment, silver trauma, end of life care in the acute setting.

Results Eight posters with expert clinical recommendations on the most important topics in Geriatric Emergency Medicine are now available through https://posters.geriemeurope.eu/.

Conclusion Expert clinical recommendations for Geriatric Emergency Medicine may help to improve care for older patients in the Emergency Department and are ready for dissemination across Europe.

Keywords Guidelines · Emergency Medicine · Geriatrics · Frailty · Acute care · Geriatric Emergency Medicine

\section{Introduction}

More older people are coming to the Emergency Department and this poses organisational challenges with longer waiting times and more physicians involved. However, we primarily notice a lack of knowledge and expertise to adequately treat older people in the ED, which leads to poor patient outcomes, such as mortality and functional decline [1-4]. Whereas medicine in general and Emergency Medicine in particular is disease-oriented and highly protocolized, older people in the Emergency Department (ED) often present with frailty syndromes or atypical complaints that may make protocol-driven approaches unsuitable. Although knowledge on frailty syndromes is increasing, it remains a challenge to translate this knowledge reliably into daily clinical care [5-7].

The European Task Force for Geriatric Emergency Medicine is a collaboration of the European Society for Emergency Medicine (EUSEM) and the European Geriatric Medicine Society (EuGMS). The Task Force has the aim to develop clinically sustainable and cost-effective, patientcentered health care systems that improve relevant outcomes for older patients in Emergency Medicine [8], upholding the principles of evidence-based medicine. Previously, a European Curriculum was composed based on which various courses have been organized to increase knowledge and experience of health care workers in Geriatric Emergency Medicine [9]. However, other parallel approaches are needed to increase impact on everyday clinical care for older ED patients throughout Europe. Developing and disseminating pragmatic guidance for Geriatric Emergency Medicine professionals, based on the latest knowledge and expert recommendations, may further help to increase knowledge and competencies of professionals and hence improve outcomes of acutely ill older patients, attempting to bridge the 'know-do gap' [10].

The aim of the present manuscript was to develop expert clinical recommendations based on expert consensus, by the review of recent guidelines and literature, on a prioritized list of topics relevant to Geriatric Emergency Medicine throughout Europe.

\section{Methods}

A three-step approach was used to develop expert clinical recommendations. We chose not to perform a series of formal systematic reviews as we anticipated they are (a) likely to deliver low-level evidence only (as recently reviewed by Preston et al. [11]), (b) with the rapid knowledge expansion likely outdated on the day of publication and (c) very time-consuming. For these reasons, we decided to make "expert clinical recommendations on Geriatric Emergency Medicine": based expert consensus and including their knowledge and review (albeit not systematic) of existing literature.

First, a modified Delphi technique was used to prioritize the most relevant topics during a face-to-face meeting in Aartselaar (Belgium) in June 2019. The expert group consisted of geriatricians, emergency physicians, nurses and researchers from eight countries, mostly in Europe. These experts were invited through the Special Interest Group on Geriatric Emergency Medicine of EuGMS and the section on Geriatric Emergency Medicine of EUSEM. 
Membership of this group was drawn from across all of Europe (list countries) and during our discussion, specific attention was paid to avoid country-specific solutions or recommendations. In addition to the topic prioritization, the meeting in Aartselaar also determined the structure and development process of the final recommendations. Second, a series of recommendations for each of the eight topics was elaborated based on literature review and expert consensus. Following the meeting, eight separate working groups worked on the content of the recommendations. Each group consisted of at least a professional with experience in Emergency Medicine and one in Geriatrics. Finally, the content of all recommendations combined were discussed in the expert group, to establish consistency, relevancy and applicability in clinical practice.

\section{Selection of topics}

A comprehensive list of topics was generated in a general discussion based on key documents in Geriatric Emergency Medicine [i.e., (1) the prioritized Research Questions (to be published), (2) the McCusker List [12], (3) the Silver Book [13], resulting in a 'long list of topics']. Next, a threeround modified Delphi procedure was used to reach consensus on the most relevant topics for which expert clinical recommendations would be written. In the first round, all attendants were asked to prioritize their top eight questions from the full list. Topics which were not included following compilation of the priority lists were eliminated. In the second round, members of the panel discussed the topics on the list and got the opportunity to justify their responses. In the second voting round, attendees received instructions to score the topics of the abbreviated list. A weighted voting system was used with each individual awarding marks to a topic based on their priority ranking. Topics were scored and tallied. A third round of discussion ensued afterwards, upon which a consensus was reached and the top prioritized topics were selected.

\section{Form of recommendations}

The expert clinical recommendations were developed in the form of posters with similar lay-out and structure across prioritized topics. The goal was to create posters that are easy to consult in the fast-paced environment of the ED, with concise and clear clinical pragmatic recommendations.

For each topic, the poster structure contained three sections addressing the following points (following the Golden Circle principle of Simon Sinek [14]):
- Why is this an important topic? e.g.: is this disease very prevalent? Is it under-recognized? Does it have a significant impact on outcomes?)

- How can we improve this situation? (For instance: does better recognition lead to better outcomes? Are there interventions that are effective to improve outcomes?)

- What can you do practically, in the form of a "Toolbox"? (For instance: what assessment tool is best to detect high risk? What intervention can you start doing in the ED now?)

All posters contain links to more elaborate overview of literature and recommendations, relevant references and the toolbox using QR-codes.

\section{Content of expert clinical recommendations}

For each topic, working groups were convened who:

- Reviewed the available evidence and sought relevant existing guidelines/reviews/landmark studies, using a PubMed search; relevant evidence was selected by the experts based on relevance for the topic.

- Formulated recommendations by the expert group, based on the literature and expert knowledge.

- Engaged relevant expertise to inform the guidance from other specialist societies, for example, the European Delirium Association and from patient groups.

The proposed content was then presented to the expert group for discussion and to reach consensus on the following:

- The content and detail of the background information provided.

- The formulation of recommendations and voting on the final recommendations. For each recommendation, con-

Table 1 Topics for Geriatric Emergency Medicine guidelines selected by experts after modified Delphi procedure

\begin{tabular}{|c|c|}
\hline Rank & Topic \\
\hline 1 & $\begin{array}{l}\text { Comprehensive Geriatric Assess- } \\
\text { ment in the Emergency Department }\end{array}$ \\
\hline 2 & Age/frailty adjusted risk stratification \\
\hline 3 & Delirium and cognitive impairment \\
\hline 4 & Family involvement \\
\hline 5 & Environment \\
\hline 6 & Polypharmacy \\
\hline 7 & Silver trauma \\
\hline 8 & End of life care in the acute setting \\
\hline
\end{tabular}


Table 2 Geriatric Emergency Medicine guidelines-recommendations per topic

Topic \# Recommendations

CGA in the ED

1.1 For older patients with frailty, Emergency Departments need to evolve from offering single problem solutions to a more holistic approach

1.2 A full CGA often cannot be implemented in the ED setting. It is important to operationalise its key concepts, such as the '5Ms of geriatrics' [30] in an initial GA:

MIND: addressing dementia, delirium and depression

MOBILITY: maintaining mobility and avoiding falls

MEDICATIONS: reducing unhelpful polypharmacy

MULTI-COMPLEXITY: addressing the multifaceted needs of older people (medical, psychological, social, functional and environmental)

MATTERS MOST: ensuring that a person's individual, personally meaningful health outcomes, goals, and care preferences are reflected in treatment plans

1.3 Then use shared decision-making to determine what the patient's priorities are. Work with your interdisciplinary team to work out how and where these can be best met (in hospital or at home or in another care facility)

Age/frailty adjusted risk stratification 2.1 Triage systems: consider incorporation of a mobility and/or frailty measure (such as CFS) in the triage system

2.2 Consider presenting complaint: non-specific complaints (feeling unwell) are associated with high in-hospital mortality

2.3 Interpretation of vital signs: check baseline values of individual patient and monitor trends. If baseline values are not available:

Systolic blood pressure: on average below 120 (in sepsis), or $110 \mathrm{mmHg}$ (in trauma) should be considered abnormal until proven otherwise

Heart rate: on average heart rate below 50 or above $100 \mathrm{bpm}$ is associated with higher in-hospital mortality and should be considered abnormal

Temperature: normal or low temperature is associated with higher mortality than high temperature in patients with suspected infection/sepsis

Look for clinical signs of organ failure: increased respiratory rate, prolonged capillary refill time, poor peripheral circulation or low urine output, altered mental status

2.4 Assess for frailty, cognitive impairment, delirium (and use in conjunction with recommendation 1 and 2)

2.5 Assess social situation and functional status and impact before hospital discharge

Delirium and cognitive impairment

3.1 Manage those patients with delirium, or at risk of developing delirium, through multicomponent interventions and regular reorientation. Perform a structured assessment including medications review that aims to identify and reverse potential causes of delirium. Checklists can be used to assist these processes

3.2 Use appropriate aids to assist assessment of pain in older people with cognitive impairment, e.g., PAINAD

3.3 Where patients have behavioral disturbance or agitation related to cognitive impairment, attempt non-pharmacological treatment methods as first line. Physical restraints should not be used

3.4 Employ a cautious approach to sedation and only use it when non-pharmacological approaches have failed. Use oral medications in the first instance with the choice of medication tailored to the individual patient. A decision to escalate to IM/IV sedation should be made by a senior doctor and administered in an area where the patient can be properly monitored and where airway support is available, following local and national sedation guidance

3.5 Link patients who are found to have cognitive impairment in the ED with local structured care pathways for inpatient care, or to their family doctor for further investigation on discharge

3.6 Tailor diagnostic investigations for delirium to the individual patient's history and physical examination findings

Family involvement

4.1 Determine the care needs of the older patient during ED admission. (Score basic and instrumental activities of daily living checklists)

4.2 Determine the level of support required by the patient in the ED and anticipate discharge needs in collaboration with the carers

4.3 When possible, assess carer burden and their capacity to carry out instructions

4.4 When discharging a patient to an outpatient setting, readily provide information about different forms of support that may be available. Ensure that your teams are able to refer patients and their carers to the necessary services that can provide or organise health and social support 
Table 2 (continued)

Topic \# Recommendations

4.5 Ensure that the patient (when possible) and their carer clearly understand arrangements related to discharge planning as well as other follow-up instructions

Environment

Polypharmacy

Trauma
4.6 Hospitals should facilitate patient and public involvement in the development of quality improvement strategies aimed at improving patients' experience in the ED

5.1 Physical space:

Small changes for immediate impact

De-clutter

Offer multiple cues for orientation to time and place, including clocks, calendars and clear wayfinding to bed spaces and toilets

Transform corridors into pleasant spaces for walking about safely

Ensure toilet seats and grab-rails are in contrasting colours

If more resources are available, install matt flooring and good-quality lighting

5.2 Equipment and human resources: ensure your older patients have easy access to mobility aids, sensory aids, continence aids and nutrition and hydration. Have staff available to facilitate access to these basic needs as well as encouraging meaningful activities

Small changes for immediate impact

Offer sensory aids_-glasses, hearing aid batteries, or a magnifying glass and portable amplifying device

Offer snacks and drinks

Encourage social dining with companions and engagement in meaningful activity in case of prolonged ED stay

5.3 Staff behaviour: staff should welcome family and ensure appropriate communication. Identifying geriatric syndromes, improving mobility and helping older people maintain their function are as important as 'traditional' emergency care

6.1 Gather as much information as possible about medication, including over-the counter medications, with a structured approach of name of drug, dose, form of application and frequency. Identify any recent changes to medications including dose alterations and enquire about compliance and concordance

6.2 Consider whether an adverse drug event could be a contributing factor for admission, taking into consideration possible drug-drug interactions and drug-disease interactions. In patients with polypharmacy, determine the anticholinergic burden as this is an important modifiable risk factor for delirium

6.3 Perform a structured and standardized screen for potentially inappropriate medications using tools such as the medication Appropriateness Index of STOPP-START

6.4 Initiate new medications cautiously and initiate deprescribing if appropriate. Consider patient's renal function and calculate the glomerular filtration rate rather than just relying solely on the creatinine. Avoid the use of analgesics such as NSAIDs or tramadol and do not use benzodiazepines as first line therapy in the management of delirium in older people

6.5 Assess patient's drug regimen taking into consideration patient preferences, life expectancy, and comorbidities. Upon discharge, ensure that the patient, their care givers and patient's health care providers are updated with the new prescription plan

6.6 If a comprehensive medication review cannot be performed in the Emergency Department, consider referring to a geriatric day hospital or clinical pharmacist for medicines optimization

7.1 Assess the older patients presenting with low energy transfer trauma for significant injuries

7.2 Aim to improve the pre-hospital and in-hospital triage systems to identify major trauma in older adults by incorporating risk stratifying tools such as silver trauma safety net and older patient trauma screening tool for trauma units, major trauma centers and local emergency hospitals

7.3 Look out for the signs of occult haemorrhage

Heart rate $>90 \mathrm{bpm}$

Systolic blood pressure $<110 \mathrm{mmHg}$

Lactate $>2.5 \mathrm{mmol} / \mathrm{L}$

7.4 Perform a CT head scan on any patient aged $\geq 65$ years with loss of consciousness or amnesia following a head injury. Furthermore, perform a CT scan on any patient with a head injury who is on warfarin, other anticoagulants (Direct Oral Anticoagulants), clopidogrel (anti-platelet therapy) or with bleeding disorders like haemophilia

7.5 Any adult $\geq 65$ years and clinical suspicion of cervical spine injury should have a CT cervical spine as soon as possible. Forced application of a collar is unnecessary as it may cause harm 
Table 2 (continued)

\begin{tabular}{llc}
\hline Topic & \# & Recommendations \\
\hline 7.6 & $\begin{array}{c}\text { The mainstay of treatment for rib fractures should be to provide adequate analgesia coupled with } \\
\text { meticulous respiratory care to prevent complications such as atelectasis and pneumonia }\end{array}$ \\
$7.7 \begin{array}{c}\text { Screen patients for underlying frailty, adapt a holistic approach to assess the multidimensional needs } \\
\text { of the individual patient and agree patient centered goals of treatment }\end{array}$ \\
$8.1 \quad \begin{array}{c}\text { Ask the patient whether they have established their goals of care with their General Practitioner or } \\
\text { organ/hospital specialist. If not: initiate such a discussion. Use positive language about need for } \\
\text { comfort care. Use optimally an opportunity of relatively compensated state of the patient }\end{array}$ \\
$8.2 \begin{array}{c}\text { Treat pain: opioids are the main tool (find out any previous use of opioids!) } \\
8.3 \text { Treat dyspnea (rule out for reversible causes like pleural effusion, fluid overload or anemia): consider } \\
\text { symptomatic relief with oxygen, opioids and/or benzodiazepines }\end{array}$ \\
$8.4 \begin{array}{c}\text { Treat nausea and vomiting: identify any reversible causes like constipation or side effects of medica- } \\
\text { tion }\end{array}$ \\
$8.5 \begin{array}{c}\text { Treat anxiety or delirium: explore possible causes (urinary retention, faecal impaction or pain). } \\
\text { Benzodiazepines or antipsychotics like haloperidol can be used }\end{array}$ \\
8.6 \begin{tabular}{c} 
GOAL: prioritize patient comfort by managing their symptoms \\
\hline
\end{tabular}
\end{tabular}

$C G A$ comprehensive geriatric assessment, $E D$ Emergency Department, $C F S$ clinical frailty score, $m m H g$ millimetres of mercury, $I V$ intravenous, $I M$ intramuscular, $C T$ computed tomography, $b m p$ beats per minute, $m m o l / L$ mill mole per litre, NSAID non-steroidal anti-inflammatory drugs

sensus was sought and found, sometimes after amendment of the recommendation.

- Signposting to specific tools.

The first versions of the posters were developed in the English language and with generic recommendations regarding specific instruments to use. The task force creating the posters was endorsed by the boards of both EUSEM and EuGMS. It is the explicit intention to translate the posters to other countries, both in language and in choice of relevant instruments. National endorsement will be sought from national organizations.

All posters will be available as PDFs and web-pages free of charge through https://posters.geriemeurope.eu/.

\section{Results}

The full list of topics contained 21 possible topics and is shown in Appendix 1. After the second round of discussion, eight topics were selected to be included in the first expert clinical recommendations and are shown in Table 1.

The process started in May 2019 and final votes on the content of the recommendations were held during an online meeting in June 2021. Over a 2-year period, two face-to-face meetings and seven online expert group meetings were held. More face to-face-meetings were planned, but cancelled due to the COVID-19 pandemic. Individual working groups for the separate topics worked in parallel.

The recommendations made by the experts on each topic can be found in Table 2 .

An example of the content of the posters is shown in Fig. 1.
Recommendations for education and implementation of the Geriatric Emergency Medicine expert clinical recommendations can be found in Appendix 2 and 3.

\section{Discussion}

This paper describes the process and outcomes of the Geriatric Emergency Medicine clinical expert group. Recommendations have been made on eight key topics. The materials to implement these recommendations into practice are provided in the appendix.

The field of Geriatric Emergency Medicine has been expanding in the last two decades. Geriatric Emergency Medicine guidelines have been developed in the USA [15]. Also, several sets of quality indicators have been published in the USA [16, 17], UK [18] and Germany [19], as well as text books that have been written on this topic [20, 21]. Furthermore, specific guidelines and reviews on typical geriatric syndromes and problems for the ED have emerged [22-24]. Our initiative is a first attempt to translate the increasing knowledge in Geriatric Emergency Medicine into guidance for professionals working in Europe. The existing lack of such translation-referred to as the 'know-do gap [10]"-hampers timely implementation of this knowledge. Because of the aim to deliver very short guidelines for clinical practice on one hand, and the expected low quantity and evidence-level of the literature [11, 25-27], we did not perform formal systematic reviews and no systematic evidencegrading, but rather used expert experience and consensus. The unmet urgent need to increase awareness, knowledge and competencies of professionals working in Geriatric Emergency Medicine validates such a pragmatic approach, 


\section{WDUSEM}

\section{AGE/FRAILTY ADJUSTED RISK STRATIFICATION}

\section{WHY IS THIS IMPORTANT?}

Older people increasingly visit the emergency department (ED), are sicker than younger patients, and have an high risk of adverse health outcomes which requires special attention to prevent further decline.

Recognition of high-risk patients is more difficult in older compared to younger patients because they present with non-specific complaints (i.e. weakness or falls), interpretation of vital signs is complicated by altered physiology, co-morbidity and polypharmacy, they are often undertriaged by frequently used triage systems, and classic risk stratification tools like NEWS have low predictive performance in older patients.

If vulnerability is recognised early, timely resuscitation, targeted interventions and disposition to the most optimal level of care, may limit the risk for adverse health outcomes, and increase awareness among ED personal to include patient's preferences in the assessment.

\section{WHICH RISKS ARE WE STRATIFYING?}

Short term: Cognitive impairment (including delirium), in-hospital mortality, unplanned avoidable re-admissions, new institutionalization to long term care facility.

Longer term: Functional decline, new institutionalisation, 3and 12-month mortality.

\section{HOW CAN WE RECOGNIZE OLDER PATIENTS AT HIGH RISK FOR ADVERSE OUTCOMES?}

Normal primary (ABCDE) assessment also applies to older patients as long as changing physiology and polypharmacy, affecting interpretation of vital signs, are taken into account.

Screening tools for frailty and/or cognitive impairment and/ or delirium that are appropriate for ED use: APOP, 6-CIT, 4-AT, Clinical Frailty Scale. See Comprehensive Geriatric Assessment (CGA) and delirium sheet.

This education material was dewelopped by the European Task Force for Geriatric Emergency Medicine, which is a collaboration between the European Society for Emergency Medicine (EUSEM) and the European Geriatric Medicine Society (EuGMS). For more information, please visit: geriEMEurope.eu and follow us on Twitter: @geriEMEurope. Download this poster via OR-code.

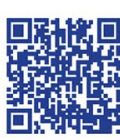

\section{WHAT CAN WE DO?}

1. Triage systems: consider incorporation of a mobility and/ or frailty measure (such as CFS) in the triage system.

2. Consider presenting complaint: non-specific complaints (feeling unwell) are associated with high in-hospital mortality.

3. Do a primary $(\mathrm{ABCDE})$ assessment. However:

\section{Interpretation of vital signs:}

Check baseline values of individual patient and 4 monitor trends. If the patient's individual baseline values are not available:

\section{Systolic blood pressure:}

On average below 120 (in sepsis), or $110 \mathrm{mmHg}$ (in trauma) should be considered abnormal until proven otherwise.

\section{Heart rate:}

On average heart rate below $\mathbf{5 0}$ or above $\mathbf{1 0 0}$ bpm is associated with higher in-hospital mortality and should be considered abnormal.

\section{Temperature:}

Normal or low temperature is associated with d. higher mortality than high temperature in patients with suspected infection/sepsis.

\section{Look for clinical signs of organ failure:} Increased respiratory rate, prolonged capillary refill time, poor peripheral circulation or low urine output, altered mental status (see below):

4. Assess for frailty, cognitive impairment, delirium (and use in conjunction with recommendation 1 and 2).

5. Assess social situation and functional status and impact before hospital discharge.

\section{TOOLBOX}

Triage or risk stratification tools potentially improving risk stratification by including frailty measures or were specifically developed in older patients in the ED: RIGAMA score, CAM-ICU, 4-AT, APOP, CFS (Clinical Frailty Scale), 6-CIT and InterRAI ED Assessment System. All toolboxes and additional information are available via $\mathrm{QR}$-code.

\section{REFERENCES}

All relevant references to scientific publications can be found via the adjacent $Q R$-code.

Fig. 1 Poster expert clinical recommendations on topic 2: age/frailty adjusted risk stratification. Example of a poster that can be used to educate caregivers in the ED on age and frailty adjusted risk stratification 
but does not at all exclude that future research should further increase evidence-level and recommendations. The expert clinical recommendations will therefore be updated regularly. Our pragmatic approach allowed us to deliver rapid guidance-although postponed by the COVID-19 pandemic - with recommendations that may have an impact on patient outcomes throughout Europe in the short term. Our recommendations are based on expert consensus and not systematic reviews. In response to the limited empirical evidence in this area, we have recently publishes a research agenda that will hopefully stimulate more research in this field [28].

\section{Dissemination across Europe}

In Europe, a large diversity in healthcare systems, available resources and existing care protocols do not allow for recommendations that are both general to all patients and specific to each healthcare setting. For instance, the presence of a strong General Practice network (which is not the case in every single country) may influence ED patient population and epidemiology as well as recommendations on post-acute care. However, with the involvement of professionals from countries across Europe we have made these expert clinical recommendations as tailored to the European situation as possible. The posters were primarily generated in English and made available to the European community of Geriatric Emergency Medicine, with the explicit intention to make them available open-access and free of charge. An example of other guidelines disseminated in this fashion are the European Resuscitation Council Guidelines [29]. In collaboration with the authors, individuals may translate the material and make changes to better suit the local national context. The posters are added in the Appendix.

\section{Implementation and education}

Recent literature indicates that ED professionals are often not well trained in geriatrics and geriatric educational programs effectively improve their knowledge and evidence-based standards of older patients care in ED [30]. Appendix 2 gives recommendations on how to implement education on Geriatric Emergency Medicine in the ED. It shows how to choose topics to be covered and discusses several different teaching options such as simulation training, microteaching and learning events. Simultaneous to educating ED staff, structural efforts need to be made to implement the clinical recommendations on Geriatric Emergency Medicine into everyday practice. Appendix 3 shows how to plan your approach and gives links to further resources. It gives advice on a multi-level and multidisciplinary approach as well as how to use data to drive change and possible barriers which can be encountered during implementation.

\section{Strengths and limitations}

One of the strengths of these expert recommendations is the use of the modified Delphi procedure, through which clinicians working in this field were able to provide input on the most important topics for which recommendations needed to be developed. The multidisciplinary and transEuropean collaboration is also a strength, with emergency physicians, geriatricians and nurses working together. The visually attractive posters that were developed, as well as an overview of the possible tools that can be applied to aid clinical decision-making, will support the education of healthcare professionals involved in the care of older people in the Emergency Department, which has to potential to improve the care for older patients throughout Europe. Using QR-codes further information can be found, which can add to the further dissemination of knowledge.

A limitation is that we did not perform a full literature review of these topics applying a GRADE procedure. Instead we used expert opinion to create expert clinical recommendations that can help closing the know-do gap. Furthermore, this is not an extensive initiative on all possible topics relevant to Geriatric Emergency Medicine. Finally, the expert group only consisted of physicians and nurses and did not include (for instance) healthcare directors or patients. We are planning however to expand the range of topics in the future and believe we covered the most important ones for this first phase.

\section{Conclusion}

Using a multidisciplinary and pan-European approach, we developed expert clinical guidelines for Geriatric Emergency Medicine. These recommendations come in visually attractive posters and advice for implementation and education, aiming to close the 'know-do' gap. Future efforts will be made to further expand these on relevant topics.

Supplementary Information The online version contains supplementary material available at https://doi.org/10.1007/s41999-021-00578-1.

Acknowledgements We would like to thank the boards of EUSEM and EuGMS for the financial support of the Task Force Clinical Expert working group. We would like to thank Dante Meeus and Willemijn van Hees for the administrative support.

Funding PH is holder of a PhD fellowship Granted by Research Foundation-Flanders (FWO) $(1133320 \mathrm{~N})$.

\section{Declarations}

Conflict of interest PH is holder of a PhD fellowship Granted by Research Foundation-Flanders (FWO) (1133320N). For the remaining authors, none were declared. 
Ethical approval No Medical Ethics Approval was sought as the study only asked physicians to voluntarily provide their professional opinion.

Informed consent No patients or patient information was involved.

\section{References}

1. Aminzadeh F, Dalziel WB (2002) Older adults in the Emergency Department: a systematic review of patterns of use, adverse outcomes, and effectiveness of interventions. Ann Emerg Med 39(3):238-247

2. Gruneir A, Silver MJ, Rochon PA (2011) Emergency Department use by older adults: a literature review on trends, appropriateness, and consequences of unmet health care needs. Med Care Res Rev 68(2):131-155

3. Schnitker L, Martin-Khan M, Beattie E, Gray L (2011) Negative health outcomes and adverse events in older people attending Emergency Departments: a systematic review. Australas Emerg Nurs 14(3):141-162

4. Heeren P et al (2021) Structure and processes of emergency observation units with a geriatric focus: a scoping review. BMC Geriatr 21(1):95

5. La Grouw Y, Bannink D, van Hout H (2020) Care professionals manage the future, frail older persons the past. Explaining why frailty management in primary care doesn't always work. Front Med (Lausanne) 7:489

6. Dent E et al (2019) Management of frailty: opportunities, challenges, and future directions. Lancet 394(10206):1376-1386

7. Hesselink $\mathrm{G}$ et al (2020) Effects of a geriatric education program for emergency physicians: a mixed-methods study. Health Educ Res 35(3):216-227

8. Mooijaart SP et al (2019) Geriatric Emergency Medicine: time for a new approach on a European level. Eur J Emerg Med 26(2):75-76

9. Bellou A, Conroy SP, Graham CA (2016) The European curriculum for Geriatric Emergency Medicine. Eur J Emerg Med 23(4):239

10. Gladman JR et al (2016) New horizons in the implementation and research of comprehensive geriatric assessment: knowing, doing and the "know-do" gap. Age Ageing 45(2):194-200

11. Preston $L$ et al (2020) Improving outcomes for older people in the Emergency Department: a review of reviews. Emerg Med J. https://doi.org/10.1136/emermed-2020-209514

12. McCusker J et al (2018) Elder-friendly Emergency Department: development and validation of a quality assessment tool. J Am Geriatr Soc 66:394-400
13. Society BG (2012) Quality care for older people with urgent and emergency care needs-'Silver Book'. BGS. https://www.bgs.org. uk/resources/resource-series/silver-book-ii

14. Sinek S (2009) Start with why. Penguin Books Ltd, London

15. American College of Emergency, P et al (2014) Geriatric Emergency Department guidelines. Ann Emerg Med 63(5):e7-e25

16. Lo AX, Biese K, Carpenter CR (2017) Defining quality and outcome in geriatric emergency care. Ann Emerg Med 70(1):107-109

17. Terrell KM et al (2009) Quality indicators for geriatric emergency care. Acad Emerg Med 16(5):441-449

18. Society BG (2021) Emergency care. Available from: https://www. bgs.org.uk/topics/emergency-care

19. Schuster $S$ et al (2020) Quality indicators for a geriatric emergency care (GeriQ-ED) — an evidence-based Delphi consensus approach to improve the care of geriatric patients in the Emergency Department. Scand J Trauma Resusc Emerg Med 28(1):68

20. Carpenter CB, Conroy SJ (2021) Silver book II. British Geriatrics Society

21. Nickel CB, Conroy A, S. (2018) Geriatric Emergency Medicine. Springer International Publishing

22. (2019) In: Delirium: prevention, diagnosis and management. London; https://www.nice.org.uk/guidance/cg 103

23. Dawood M (2020) End of life care in the Emergency Department. Emerg Med J 37(5):273-278

24. Carpenter CR et al (2019) Older adult falls in Emergency Medicine: 2019 update. Clin Geriatr Med 35(2):205-219

25. Hanning J et al (2019) Review article: goals-of-care discussions for adult patients nearing end of life in Emergency Departments: a systematic review. Emerg Med Australas 31(4):525-532

26. Turner J et al (2015) In: What evidence is there on the effectiveness of different models of delivering urgent care? A rapid review. Southampton (UK): NIHR Journals Library; 2015 Nov

27. Crede SH et al (2017) What is the evidence for the management of patients along the pathway from the Emergency Department to acute admission to reduce unplanned attendance and admission? An evidence synthesis. BMC Health Serv Res 17(1):355

28. Mooijaart SP et al (2020) A European Research Agenda for Geriatric Emergency Medicine: a modified Delphi study. Eur Geriatr Med 12:413-422

29. Council ER (2021) ERC guidelines. Available from: https://cprgu idelines.eu/\#

30. Ringer T et al (2018) White paper-Geriatric Emergency Medicine education: current state, challenges, and recommendations to enhance the emergency care of older adults. AEM Educ Train 2(Suppl 1):S5-S16

Publisher's Note Springer Nature remains neutral with regard to jurisdictional claims in published maps and institutional affiliations. 